Dept. of Math. University of Oslo

PuRe Mathematics

$\begin{array}{lr}\text { ISBN 82-553-1401-6 } & \text { No. } 31 \\ \text { ISSN 0806-2439 } & \text { SEPTEMBER 2003 }\end{array}$

\title{
Partial observation control in an anticipating environment
}

\author{
Bernt $\varnothing \mathrm{ksendal}^{1), 2)}$ and Agnès Sulem ${ }^{3)}$
}

June 10, 2004

1) Center of Mathematics for Applications (CMA)

Department of Mathematics, University of Oslo

P.O. Box 1053 Blindern, N-0316, Oslo, Norway

Email: oksendal@math.uio.no

and

2) Norwegian School of Economics and Business Administration, Helleveien 30, N-5045, Bergen, Norway

3) INRIA, Domaine de Voluceau, Rocquencourt, B.P. 105,

F-78153 Le Chesnay Cedex, France

Email: agnes.sulem@inria.fr

\begin{abstract}
We study a controlled stochastic system whose state $X(t)$ at time $t$ is described by a stochastic differential equation driven by Lévy processes with filtration $\left\{\mathcal{F}_{t}\right\}_{t \in[0, T]}$. The system is anticipating, in the sense that the coefficients are assumed to be adapted to a filtration $\left\{\mathcal{G}_{t}\right\}_{t \geq 0}$, where $\mathcal{F}_{t} \subseteq \mathcal{G}_{t}$ for all $t \in[0, T]$. The corresponding anticipating stochastic differential equation is interpreted in the sense of forward integrals, which are the natural generalization of the semimartingale integrals.

The admissible controls are assumed to be adapted to a filtration $\left\{\mathcal{E}_{t}\right\}_{t \in[0, T]}$, such that $\mathcal{E}_{t} \subseteq \mathcal{F}_{t}$ for all $t \in[0, T]$. The general problem is to maximize a given performance functional of this system over all admissible controls. This is a partial observation stochastic control problem in an anticipating environment. Examples of applications include stochastic volatity models in finance, insider influenced financial markets and stochastic control of systems with delayed noise effects.

Some specific cases from finance, involving optimal portfolio with logarithmic utility, are solved explicitly.
\end{abstract}

MSC (2000): 93E20, 60G51, 60H07, 60H30, 91B28.

Key words: Anticipating systems, partial observation control, forward integrals, Malliavin calculus. 


\section{Introduction}

Let $B(t)=\left(B_{1}(t), \ldots, B_{m}(t)\right)$ and $\eta(t)=\left(\eta_{1}(t), \ldots, \eta_{\ell}(t)\right)$ be (independent) $m$-dimensional Brownian motion and $\ell$-dimensional Lévy process, respectively, on a filtered probability space $\left(\Omega, \mathcal{F},\left\{\mathcal{F}_{t}\right\}_{t>0}, P\right)$. Assume that $E\left[\eta^{2}(t)\right]<\infty$ (where $E$ denotes expectation with respect to $P)$ and let $\tilde{N}(d t, d z)=\left(\tilde{N}_{1}\left(d t, d z_{1}\right), \ldots, \tilde{N}_{\ell}\left(d t, d z_{\ell}\right)\right), z=\left(z_{1}, \ldots, z_{\ell}\right)$, be the corresponding compensated Poisson random measure.

Let $\left\{\mathcal{E}_{t}\right\}_{t \geq 0}$ and $\left\{\mathcal{G}_{t}\right\}_{t \geq 0}$ be two filtrations such that

$$
\mathcal{E}_{t} \subseteq \mathcal{F}_{t} \subseteq \mathcal{G}_{t} \subseteq \mathcal{F} \quad \text { for all } t \geq 0
$$

We consider a controlled stochastic system whose state $X^{(u)}(t)=X(t)=\left(X_{1}(t), \ldots, X_{n}(t)\right)$ at time $t \in[0, T]$ is described by a stochastic differential equation of the form

$$
\begin{aligned}
d X(t)=b(t, X(t), u(t), \omega) d t+\sigma(t, X(t), u(t), \omega) d^{-} B(t) & \\
& +\int_{\mathbb{R}^{\ell}} \theta(t, X(t), u(t), z, \omega) \tilde{N}\left(d^{-} t, d z\right) ; \quad X(0)=x \in \mathbb{R}^{n},
\end{aligned}
$$

i.e.

$$
\begin{aligned}
X(t)=X(0) & +\int_{0}^{t} b(s, X(s), u(s), \omega) d s+\int_{0}^{t} \sigma(s, X(s), u(s), \omega) d^{-} B(s) \\
& +\int_{0}^{t} \int_{\mathbb{R}^{\ell}} \theta\left(s, X\left(s^{-}\right), u\left(s^{-}\right), z, \omega\right) \tilde{N}\left(d^{-} s, d z\right),
\end{aligned}
$$

where $b:[0, T] \times \mathbb{R}^{n} \times K \times \Omega \rightarrow \mathbb{R}^{n}, \sigma:[0, T] \times \mathbb{R}^{n} \times K \times \Omega \rightarrow \mathbb{R}^{n \times m}$ and $\theta:[0, T] \times \mathbb{R}^{n} \times$ $K \times \mathbb{R}^{\ell} \times \Omega \rightarrow \mathbb{R}^{n \times \ell}$ are given functions, $K \subset \mathbb{R}^{k}$ is a given set of admissible control values and our control process $u(t)=u(t, \omega) \in K$ is assumed to be adapted to the filtration $\left\{\mathcal{E}_{t}\right\}_{t \geq 0}$.

We assume that for each given $x \in \mathbb{R}^{n}, v \in K$ and $z \in \mathbb{R}^{\ell}$ the random variables

$$
b(t, x, v, \cdot), \sigma(t, v, v, \cdot) \text { and } \theta(t, x, v, z, \cdot) \text { are } \mathcal{G}_{t} \text {-measurable. }
$$

In other words, $b, \sigma$ and $\theta$ are assumed to be adapted to the filtration $\left\{\mathcal{G}_{t}\right\}_{t \geq 0}$. Since $B(t)$ and $\eta(t)$ need not be semimartingales with respect to $\left\{\mathcal{G}_{t}\right\}_{t \geq 0}$, the last two integrals in (1.2) are anticipating stochastic integrals. We choose to interpret these integrals as forward integrals (denoted by $d^{-} B(t)$ and $\tilde{N}\left(d^{-} t, d z\right)$, respectively), because this is what the integrals would be identical to if we happen to be in a semimartingale context. (See Lemma 2.8b) and Lemma 3.8.)

Let $f:[0, T] \times \mathbb{R}^{n} \times K \rightarrow \mathbb{R}$ and $g: \mathbb{R}^{n} \rightarrow \mathbb{R}$ be given functions and let $\mathcal{A}_{\mathcal{E}}$ be a given set of admissible controls contained in the set of $\mathcal{E}_{t}$-adapted processes $u(t)$ such that (1.2) has a strong $\mathcal{G}_{t^{-}}$adaped solution $X(t)=X^{(u)}(t)$ and such that

$$
J^{(u)}(x)=E^{x}\left[\int_{0}^{T} f(t, X(t), u(t)) d t+g(X(T))\right]
$$


converges. We consider the following problem of partial observation control in an anticipating environment:

Problem 1.1 Find $\Phi(x)$ and $u^{*} \in \mathcal{A}_{\mathcal{E}}$ such that

$$
\Phi(x)=\sup _{u \in \mathcal{A}_{\mathcal{E}}} J^{(u)}(x)=J^{\left(u^{*}\right)}(x) .
$$

This type of problem appears in many situations. We give 3 examples from mathematical finance:

\section{Example 1.2 (Stochastic volatility models)}

Suppose we have a market with one risky investment possibility (e.g. a stock), whose price $S_{1}(t)$ at time $t$ is described by a stochastic differential equation of the form

$$
d S_{1}(t)=S_{1}\left(t^{-}\right)\left[\mu(t) d t+\sigma(t) d^{-} B(t)+\int_{\mathbb{R}} \theta(t, z) \tilde{N}\left(d^{-} t, d z\right)\right],
$$

where $B$ and $\tilde{N}$ are 1-dimensional (for simplicity). In general stochastic volatility models the coefficient $\sigma(t)=\sigma(t, \omega)$ need not be $\mathcal{F}_{t}$-adapted, but can possibly be influenced by other noises as well. So the $\sigma$-algebra $\mathcal{G}_{t}$ generated by $\{\sigma(s, \cdot) ; s \leq t\}$ may be bigger than $\mathcal{F}_{t}$. The same may apply to $\theta(t, z)=\theta(t, z, \omega)$ and to $\mu(t)=\mu(t, \omega)$.

Suppose the market also has a risk free investment possibility, where the price $S_{0}(t)$ at time $t$ is described by

$$
d S_{0}(t)=\rho(t) S_{0}(t) d t ; \quad S_{0}(0)=1
$$

where $\rho(t)=\rho(t, \omega)$ is another $\mathcal{G}_{t}$-adapted process. A portfolio $\pi(t)=\pi(t, \omega)$ in this market is an $\mathcal{E}_{t}$-adapted process giving the fraction of the total wealth $X(t)$ of an agent invested in the risky asset at time $t$. The dynamics of the wealth process $X(t)=X^{(\pi)}(t)$ corresponding to the portfolio $\pi$ is then found as follows:

$$
\begin{aligned}
d X(t)=X\left(t^{-}\right)\left[(\rho(t)+(\mu(t)-\rho(t)) \pi(t)) d t+\pi(t) \sigma(t) d^{-} B(t)\right. & \\
& +\pi(t) \int_{\mathbb{R}} \theta(t, z) \tilde{N}\left(d^{-} t, d z\right] ; \quad X(0)=x>0 .
\end{aligned}
$$

The requirement that $\pi(t)$ be $\mathcal{E}_{t^{-}}$adapted models the situation that the agent only has partial information (less than $\mathcal{F}_{t}$ ) to her disposal when making the portfolio decisions. The optimal portfolio problem of the agent is to find $\Phi(x)$ and $\pi^{*} \in \mathcal{A}_{\mathcal{E}}$ such that

$$
\Phi(x)=\sup _{\pi \in \mathcal{A}_{\mathcal{E}}} E^{x}\left[U\left(X^{(\pi)}(T)\right)\right]
$$

where $U: \mathbb{R} \rightarrow[-\infty, \infty)$ is a given utility function. 


\section{Example 1.3 (Insider influenced markets)}

Consider again the market (1.6)-(1.7). If there are large investors in the market and these investors have inside information, this means that they have access to a larger filtration $\mathcal{G}_{t} \supset \mathcal{F}_{t}$ when making their decisions. This leads to a price dynamics where the coefficients $\rho(t), \mu(t), \sigma(t)$ and $\theta(t, z)$ are $\mathcal{G}_{t}$-measurable and not necessarily $\mathcal{F}_{t}$-measurable. A partially informed investor in this market will again face a problem of the form (1.8)-(1.9).

\section{Example 1.4 (Markets with delayed effects from the noise)}

Suppose we have a market with no jumps $(\theta=0)$ and with stock prices $S_{1}(t), \ldots, S_{N}(t)$ given by

$$
d^{-} S_{i}(t)=S_{i}(t)\left[\mu_{i}(t) d t+\sum_{j=1}^{N} \sigma_{i j}(t) d^{-} B_{j}\left(t-\delta_{i}\right)\right] ; \quad 1 \leq i \leq N .
$$

As before $B(t)=\left(B_{1}(t), \ldots, B_{N}(t)\right)$ is an $N$-dimensional Brownian motion with filtration $\mathcal{F}_{t}$. We asume that $\mu_{i}(t)$ and $\sigma_{i j}(t)$ are $\mathcal{F}_{t}$-adapted. However, in this model we allow for a delay $\delta_{i} \geq 0$ in the effect on $S_{i}(\cdot)$ of the noise coming from $B(\cdot)$. Moreover, for some of the stocks the effect of the same underlying noise may come later than for others, so the $\delta_{i}$ 's need not be the same.

Integrating (1.10) we get

$$
\begin{aligned}
S_{i}(t) & =S_{i}(0)+\int_{0}^{t} S_{i}(s) \mu_{i}(s) d s+\sum_{j=1}^{N} \int_{0}^{t} S_{i}(s) \sigma_{i j}(s) d^{-} B_{j}\left(s-\delta_{i}\right) \\
& =S_{i}(0)+\int_{-\delta_{i}}^{t-\delta_{i}} S_{i}\left(r+\delta_{i}\right) \mu_{i}\left(r+\delta_{i}\right) d r+\sum_{j=1}^{N} \int_{-\delta_{i}}^{t-\delta_{i}} S_{i}\left(r+\delta_{i}\right) \sigma_{i j}\left(r+\delta_{i}\right) d^{-} B_{j}(r) .
\end{aligned}
$$

Define

$$
\tilde{S}_{i}(t)=S_{i}\left(t+\delta_{i}\right) ; \quad-\delta_{i} \leq t ; \quad 1 \leq i \leq N .
$$

Then (1.11) can be written

$$
\begin{aligned}
\tilde{S}_{i}(t) & =S_{i}(0)+\int_{-\delta_{i}}^{t} \tilde{S}_{i}(r) \mu_{i}\left(r+\delta_{i}\right) d r+\sum_{j=1}^{N} \int_{-\delta_{i}}^{t} \tilde{S}_{i}(r) \sigma_{i j}\left(r+\delta_{i}\right) d^{-} B_{j}(r) \\
& =\tilde{S}_{i}(0)+\int_{0}^{t} \tilde{S}_{i}(r) \mu_{i}\left(r+\delta_{i}\right) d r+\sum_{j=1}^{N} \int_{0}^{t} \tilde{S}_{i}(r) \sigma_{i j}\left(r+\delta_{i}\right) d^{-} B_{j}(r) .
\end{aligned}
$$

Or, equivalently,

$$
d \tilde{S}_{i}(t)=\tilde{S}_{i}(t)\left[\tilde{\mu}_{i}(t) d t+\sum_{j=1}^{N} \tilde{\sigma}_{i j}(t) d^{-} B_{j}(t)\right] ; \quad \tilde{S}_{i}(0)=S_{i}(\delta) ; \quad 1 \leq i \leq N
$$


where $\tilde{\mu}_{i}(t)=\mu_{i}\left(t+\delta_{i}\right), \tilde{\sigma}_{i j}(t)=\sigma_{i j}\left(t+\delta_{i}\right) ; i \leq i, j \leq N$.

Note that this is a price equation of the same type as in (1.6) (Example 1.2), where the coefficients $\tilde{\mu}_{i}(t), \tilde{\sigma}_{i j}(t)$ are adapted to the filtration

$$
\mathcal{G}_{t}:=\mathcal{F}_{t+\delta},
$$

where

$$
\delta=\max \left(\delta_{1}, \ldots, \delta_{N}\right) .
$$

Again we may now consider an optimal portfolio problem of the form (1.9), where the information available to the agent is modelled by some given filtration $\mathcal{E}_{t} \subseteq \mathcal{F}_{t}$.

The purpose of this paper is to give an explicit solution of the problem type described in Example 1.2 in the logarithmic utility case, i.e. when

$$
U(x)=\log x ; \quad x>0 .
$$

For simplicity we will split the discussion into two cases:

(i) The continuous case $(\sigma \neq 0, \theta=0)$

(ii) The pure jump case $(\sigma=0, \theta \neq 0)$

\section{The continuous case $(\theta=0)$}

Referring to Examples 1.2 and 1.3 we now study the market $\mathcal{M}(\mathcal{E}, \mathcal{G})$ given by

$$
\begin{array}{lll}
\text { (bond price) } & d S_{0}(t)=\rho(t) S_{0}(t) d t ; \quad S_{0}(0)=1 \\
\text { (stock price) } & d S_{1}(t)=S_{1}(t)\left[\mu(t) d t+\sigma(t) d^{-} B(t)\right] ; & S_{1}(0)>0
\end{array}
$$

where we assume that $\rho(t), \mu(t)$ and $\sigma(t)$ satisfy the following conditions:

$$
\rho(t), \mu(t), \sigma(t) \quad \text { are } \mathcal{G}_{t} \text {-adapted (see (1.3)) }
$$

$$
E\left[\int_{0}^{T}\left\{|\rho(t)|+|\mu(t)|+\sigma^{2}(t)\right\} d t\right]<\infty
$$

$\sigma(t)$ is Malliavin differentiable and $D_{t^{+}} \sigma(t)=\lim _{s \rightarrow t^{+}} D_{s} \sigma(t) \quad$ exists

for a.a. $t \in[0, T]$ where $D_{s}$ denotes the Malliavin derivative at $s$ (see Definition 2.5 below).

As before $\left\{\mathcal{E}_{t}\right\}_{t \in[0, T]}$ and $\left\{\mathcal{G}_{t}\right\}_{t \in[0, T]}$ are given filtrations such that

$$
\mathcal{E}_{t} \subseteq \mathcal{F}_{t} \subseteq \mathcal{G}_{t} \subseteq \mathcal{F} \quad \text { for all } t \in[0, T]
$$


Definition 2.1 The set $\mathcal{A}_{\mathcal{E}}$ of admissible portfolios consists of all processes $\pi(t)$ satisfying the following conditions:

$$
\begin{aligned}
& \pi(t) \quad \text { is } \mathcal{E}_{t^{-} \text {-adapted }} \\
& \pi(t) \sigma(t) \quad \text { is Skorohod integrable and caglad (i.e. left continuous with } \\
& \text { existing right hand side limit) } \\
& E\left[\int_{0}^{T}\left|\pi(t) D_{t^{+}} \sigma(t)\right| d t\right]<\infty \\
& E\left[\int_{0}^{T}|\mu(t)-\rho(t)| \cdot|\pi(t)| d t\right]<\infty .
\end{aligned}
$$

Referring to Example 1.2 we study the following partial observation optimal portfolio problem:

Problem 2.2 Find $\Phi(x)$ and $\pi^{*} \in \mathcal{A}_{\mathcal{E}}$ such that

$$
\Phi(x)=\sup _{\pi \in \mathcal{A}_{\mathcal{E}}} E^{x}\left[\log \left(X^{(\pi)}(T)\right)\right]=E^{x}\left[\log \left(X^{\left(\pi^{*}\right)}(T)\right)\right]
$$

where $X^{(\pi)}(t)=X(t)$ is given by $X(0)=x>0$ and

$$
d X(t)=X(t)\left[(\rho(t)+(\mu(t)-\rho(t)) \pi(t)) d t+\pi(t) \sigma(t) d^{-} B(t)\right] .
$$

The function $\Phi \leq \infty$ is called the value function and $\pi^{*}$ (if it exists) is called an optimal portfolio for Problem 2.2.

Before solving Problem 2.2 we review for the convenience of the reader some basic mathematical background. We refer to $[\mathrm{NP}],[\mathrm{N}]$ and $[\varnothing]$ for more details.

Let $\lambda$ denote Lebesgue measure on $[0, T]$ and let $L^{2}\left(\lambda^{n}\right)$ be the space of all deterministic functions $f:[0, T]^{n} \rightarrow \mathbb{R}$ such that

$$
\|f\|_{L^{2}\left(\lambda^{n}\right)}=\int_{[0, T]^{n}} f^{2}(x) d \lambda(x)=\int_{[0, T]^{n}} f^{2}\left(x_{1}, \ldots, x_{n}\right) d x_{1} \ldots d x_{n}<\infty .
$$

If $f$ is a real function on $[0, T]^{n}$ we define its symmetrization $\tilde{f}$ by

$$
\tilde{f}\left(t_{1}, \ldots, t_{n}\right)=\frac{1}{n !} \sum_{\alpha} f\left(t_{\alpha_{1}}, \ldots, t_{\alpha_{n}}\right)
$$

where the sum is taken over all permutations $\alpha$ of $\{1,2, \ldots, n\}$. We say that $f$ is symmetric if $\tilde{f}=f$ and we let $\tilde{L}^{2}\left(\lambda^{n}\right)$ denote the set of all symmetric functions in $L^{2}\left(\lambda^{n}\right)$. Put

$$
\mathcal{S}_{n}=\left\{\left(t_{1}, \ldots, t_{n}\right) \in[0, T]^{n} ; 0 \leq t_{1} \leq \cdots \leq t_{n} \leq T\right\}
$$


If $f \in L^{2}\left(\mathcal{S}_{n}\right)$ we define its $n$-fold iterated integral with respect to $B(\cdot)$ by

$$
J_{n}(f)=\int_{0}^{T} \int_{0}^{t_{n}} \ldots \int_{0}^{t_{2}} f\left(t_{1}, \ldots, t_{n}\right) d B\left(t_{1}\right) \ldots d B\left(t_{n}\right)
$$

and if $f \in \tilde{L}^{2}\left(\lambda^{n}\right)$ we define

$$
I_{n}(f):=\int_{[0, T]^{n}} f\left(t_{1}, \ldots, t_{n}\right) d B^{\otimes n}(t):=n ! J_{n}(f) .
$$

We can now formulate the Wiener-Itô chaos expansion theorem:

Theorem 2.3 Every $\mathcal{F}_{T}$-measurable random variable $F \in L^{2}(P)$ can be written

$$
F=E[F]+\sum_{n=1}^{\infty} I_{n}\left(f_{n}\right):=\sum_{n=0}^{\infty} I_{n}\left(f_{n}\right)
$$

for a unique sequence of deterministic functions $f_{n} \in \tilde{L}^{2}\left(\lambda^{n}\right)$. Moreover, we have the isometry

$$
E\left[F^{2}\right]=(E[F])^{2}+\sum_{n=1}^{\infty} n !\left\|f_{n}\right\|_{L^{2}\left(\lambda^{n}\right)}^{2} .
$$

This expansion is useful for the definition of Skorohod integrals and Malliavin derivatives: Let $\phi(t, \omega):[0, T] \times \Omega \rightarrow \mathbb{R}$ be a measurable process such that

$$
E\left[\phi^{2}(t, \cdot)\right]<\infty
$$

and

$$
\phi(t, \cdot) \quad \text { is } \mathcal{F}_{T} \text {-measurable for all } t \in[0, T] .
$$

Let

$$
\phi(t, \omega)=\sum_{n=0}^{\infty} I_{n}\left(f_{n}(\cdot, t)\right)
$$

be the chaos expansion of $\phi(t, \cdot)$ and let $\tilde{f}\left(t_{1}, \ldots, t_{n}, t_{n+1}\right)$ be the symmetrization of $f\left(t_{1}, \ldots\right.$, $\left.t_{n}, t\right)$ with respect to the $n+1$ variables $t_{1}, \ldots, t_{n}, t_{n+1}=t$.

Definition 2.4 Suppose that

$$
\sum_{n=0}^{\infty}(n+1) !\left\|\tilde{f}_{n}\right\|_{L^{2}\left(\lambda^{n+1}\right)}^{2}<\infty .
$$

Then we define the Skorohod integral of $\phi$ with respect to $B(\cdot)$ by

$$
\int_{0}^{T} \phi(t, \omega) \delta B(t)=\sum_{n=0}^{\infty} I_{n+1}\left(\tilde{f}_{n}\right)
$$


Note that by (2.14) we have

$$
E\left[\left(\int_{0}^{T} \phi(t, \omega) \delta B(t)\right)^{2}\right]=\sum_{n=0}^{\infty}(n+1) !\left\|\tilde{f}_{n}\right\|_{L^{2}\left(\lambda^{n+1}\right)}^{2}<\infty
$$

so the Skorohod integral belongs to $L^{2}(P)$ when defined. Moreover,

$$
E\left[\int_{0}^{T} \phi(t, \omega) \delta B(t)\right]=0
$$

One can show that the Skorohod integral is an extension of the Itô integral, in the sense that if $\phi(t, \omega)$ is $\mathcal{F}_{t}$-adapted and Skorohod integrable then

$$
\int_{0}^{T} \phi(t, \omega) \delta B(t)=\int_{0}^{T} \phi(t, \omega) d B(t) .
$$

Definition 2.5 Let $F \in L^{2}(P)$ be $\mathcal{F}_{T}$-measurable, with the expansion

$$
F=\sum_{n=0}^{\infty} I_{n}\left(f_{n}\right) ; \quad f_{n} \in \tilde{L}^{2}\left(\lambda^{n}\right)
$$

We say that $F$ is Malliavin differentiable and write $F \in \mathbb{D}_{1,2}$ if

$$
\|F\|_{\mathbb{D}_{1,2}}^{2}:=(E[F])^{2}+\sum_{n=1}^{\infty} n n !\left\|f_{n}\right\|_{L^{2}\left(\lambda^{n}\right)}^{2}<\infty .
$$

If $F \in \mathbb{D}_{1,2}$ we define the Malliavin derivative of $F$ at $t \in[0, T]$ by

$$
D_{t} F=\sum_{n=1}^{\infty} n I_{n-1}\left(f_{n}(\cdot, t)\right)
$$

where $I_{n-1}\left(f_{n}(\cdot, t)\right)$ is the $n-1$ iterated integral of $f\left(t_{1}, \ldots, t_{n-1}, t\right)$ as a function of the $n-1$ first variables $t_{1}, \ldots, t_{n-1}$.

Since

$$
E\left[\int_{0}^{T}\left(D_{t} F\right)^{2} d t\right]=\sum_{n=1}^{\infty} n n !\left\|f_{n}\right\|_{L^{2}\left(\lambda^{n}\right)}^{2}
$$

we see that if (2.18) holds then $D_{t} F$ exists for a.a. $t \in[0, T]$.

The Malliavin derivative $D_{t}$ satisfies the usual chain rule. For example, we have: 
Lemma 2.6 Let $f \in C^{1}(\mathbb{R})$ with bounded derivatives and let $F \in \mathbb{D}_{1,2}$. Then $f(F) \in \mathbb{D}_{1,2}$ and

$$
D_{t}(f(F))=f^{\prime}(F) \cdot D_{t} F
$$

Next we recall the definition of forward integrals with respect to $B(\cdot)$. We refer to $[\mathrm{NP}]$, [RV1] and [RV2] for more information about these integrals.

Definition 2.7 Let $\phi:[0, T] \times \Omega \rightarrow \mathbb{R}$ be a measurable process, not necessarily $\mathcal{F}_{t}$-adapted. Then we define the forward integral of $\phi$ with respect to $B(\cdot)$ by

$$
\int_{0}^{T} \phi(t) d^{-} B(t)=\lim _{\epsilon \rightarrow 0} \int_{0}^{T} \phi(t) \frac{B(t+\epsilon)-B(t)}{\epsilon} d t
$$

if the limit exists in probability.

By using a stochastic Fubini theorem we obtain the following more suggestive description of the forward integral:

Lemma 2.8 a) Let $\phi:[0, T] \times \Omega \rightarrow \mathbb{R}$ be forward integrable and caglad. Then

$$
\int_{0}^{T} \phi(t) d^{-} B(t)=\lim _{\Delta t \rightarrow 0} \sum_{j=0}^{N-1} \phi\left(t_{j}\right)\left(B\left(t_{j+1}\right)-B\left(t_{j}\right)\right)
$$

(limit in probability), where $0=t_{0}<t_{1}<\cdots<t_{N}=T$ is a partition of $[0, T]$ and $\Delta t=t_{j+1}-t_{j}$ for all $j=0, \ldots, N-1$.

b) Suppose in addition that $B(t)$ is a semimartingale with respect to $\mathcal{G}_{t}$, and that $\phi(t)$ is $\mathcal{G}_{t}$-measurable for all $t \in[0, T]$. Then

$$
\int_{0}^{T} \phi(t) d^{-} B(t)=\int_{0}^{T} \phi(t) d B(t)
$$

where the integral on the right is the usual (semimartingale) Itô integral.

ProOF. This well-known result follows by the same argument as in [BØ, (2.2) and Corollary 2.5].

A proof of the following basic relation between the forward integral and the Skorohod integral can be found in [BØ, Lemma 2.2]: 
Lemma 2.9 Suppose $\phi:[0, T] \times \Omega \rightarrow \mathbb{R}$ is Skorohod integrable and caglad. Moreover, assume that

$$
D_{t^{+}} \phi(t):=\lim _{s \rightarrow t^{+}} D_{s} \phi(t)
$$

exists for a.a. $t \in[0, T]$ and

$$
\int_{0}^{T}\left|D_{t^{+}} \phi(t)\right| d t<\infty .
$$

Then the forward integral of $\phi$ exists and

$$
\int_{0}^{T} \phi(t) d^{-} B(t)=\int_{0}^{T} \phi(t) \delta B(t)+\int_{0}^{T} D_{t^{+}} \phi(t) d t .
$$

Since Skorohod integrals have expectation 0 (see (2.17)) we deduce from Lemma 2.9 the following

Corollary 2.10 Let $\phi$ be as in Lemma 2.9. Then

$$
E\left[\int_{0}^{T} \phi(t) d^{-} B(t)\right]=E\left[\int_{0}^{T} D_{t^{+}} \phi(t) d t\right],
$$

provided that the expectations exist.

We also need the following Itô formula for forward integrals:

Theorem 2.11 ([RV2]) Let $X(t)$ be a stochastic process of the form

$$
X(t)=X(0)+\int_{0}^{t} \alpha(s) d s+\int_{0}^{t} \gamma(s) d^{-} B(s) .
$$

Let $f \in C^{1,2}\left(\mathbb{R}^{2}\right)$ and define

$$
Y(t)=f(t, X(t))
$$

Then

$$
\begin{aligned}
Y(t)=Y(0) & +\int_{0}^{t} \frac{\partial f}{\partial s}(s, X(s)) d s+\int_{0}^{t} \frac{\partial f}{\partial x}(s, X(s)) d^{-} X(s) \\
& +\frac{1}{2} \int_{0}^{t} \frac{\partial^{2} f}{\partial x^{2}}(s, X(s)) \gamma^{2}(s) d s
\end{aligned}
$$

where

$$
d^{-} X(s)=\alpha(s) d s+\gamma(s) d^{-} B(s)
$$


We now proceed to solve Problem 2.2:

Applying Theorem 2.11 to the forward equation (2.13) we get the (unique) solution

$$
\begin{array}{r}
X^{(\pi)}(T)=x \exp \left\{\int_{0}^{T}(\rho(t)+(\mu(t)-\rho(t)) \pi(t)\right. \\
\left.\left.-\frac{1}{2} \pi^{2}(t) \sigma^{2}(t)\right) d t+\int_{0}^{T} \pi(t) \sigma(t) d^{-} B(t)\right\} .
\end{array}
$$

Hence, using (2.24),

$$
\begin{aligned}
E & {\left[\log X^{(\pi)}(T)\right]-\log x=} \\
& =E\left[\int_{0}^{T}\left(\rho(t)+(\mu(t)-\rho(t)) \pi(t)-\frac{1}{2} \pi^{2}(t) \sigma^{2}(t)\right) d t+\int_{0}^{T} \pi(t) \sigma(t) d^{-} B(t)\right] \\
& =E\left[\int_{0}^{T}\left\{\rho(t)+(\mu(t)-\rho(t)) \pi(t)-\frac{1}{2} \pi^{2}(t) \sigma^{2}(t)+D_{t^{+}}(\pi(t) \sigma(t))\right\} d t\right] .
\end{aligned}
$$

Since $\pi(t)$ is $\mathcal{E}_{t}$-measurable and $\mathcal{E}_{t} \subseteq \mathcal{F}_{t}$ we have

$$
D_{s} \pi(t)=0 \quad \text { for all } s>t .
$$

Therefore, by the chain rule for the Malliavin derivative

$$
D_{t^{+}}(\pi(t) \sigma(t))=\sigma(t) D_{t^{+}} \pi(t)+\pi(t) D_{t^{+}} \sigma(t)=\pi(t) D_{t^{+}} \sigma(t),
$$

which substituted into (2.27) gives

$$
E\left[\log X^{(\pi)}(T)\right]-\log x=E\left[\int_{0}^{T}\left\{\rho(s)+\beta(s) \pi(s)-\frac{1}{2} \sigma^{2}(s) \pi^{2}(s)\right\} d s\right],
$$

where

$$
\beta(s):=\mu(s)-\rho(s)+D_{s^{+}} \sigma(s) .
$$

Equation (2.31) can also be written

$$
E\left[\log X^{(\pi)}(T)\right]-\log x=E\left[\int_{0}^{T}\left\{\hat{\rho}(s)+\hat{\beta}(s) \pi(s)-\frac{1}{2} \hat{\sigma}^{2}(s) \pi^{2}(s)\right\} d s\right],
$$

where

$$
\hat{\rho}(s)=E\left[\rho(s) \mid \mathcal{E}_{s}\right]
$$


and similarly for $\hat{\sigma}, \hat{\beta}, \hat{\sigma}^{2}$. We can now maximise poinwise for each $s$ with respect to $\pi$ under the integral sign. We obtain:

$$
\pi^{*}(s) \hat{\sigma^{2}}(s)=\hat{\beta}(s) .
$$

Summarizing the above we get the following result:

Theorem 2.12 a) Suppose that $\sigma(t) \neq 0$ for a.a. $(t, \omega)$ and

$$
E\left[\int_{0}^{T} \frac{\hat{\beta}^{2}(s)}{\hat{\sigma}^{2}(s)} d s\right]<\infty
$$

where $\beta(s)$ is defined in (2.29). Then the value function $\Phi$ of Problem 2.2 is

$$
\Phi(x)=\log x+E\left[\int_{0}^{T}\left\{\rho(s)+\frac{\beta(s) \hat{\beta}(s)}{\hat{\sigma}^{2}(s)}-\frac{\sigma^{2}(s)}{2}\left(\frac{\hat{\beta}(s)}{\hat{\sigma}^{2}(s)}\right)^{2}\right\} d s\right] .
$$

It is also equal to

$$
\Phi(x)=\log x+E\left[\int_{0}^{T}\left\{\rho(s)+\frac{\hat{\beta}(s)^{2}}{2 \hat{\sigma}^{2}(s)}\right\} d s\right]<\infty .
$$

b) Suppose that $\sigma(t) \neq 0$ for a.a. $(t, \omega)$ and that

$$
\hat{\pi}(s):=\frac{\hat{\beta}(s)}{\hat{\sigma^{2}}(s)} \in \mathcal{A}_{\mathcal{E}} .
$$

Then $\pi^{*}(s):=\hat{\pi}(s)$ is an optimal control for Problem 2.2.

c) Suppose there exists an optimal portfolio $\pi^{*} \in \mathcal{A}_{\mathcal{E}}$ for Problem 2.2. Then

$$
\pi^{*}(s) \hat{\sigma^{2}}(s)=\hat{\beta}(s)
$$

Corollary 2.13 a) Suppose

$$
\sigma(s) \quad \text { is } \mathcal{F}_{s} \text {-measurable for all } s \in[0, T] \text {. }
$$

Then

$$
D_{s+} \sigma(s)=0 \quad \text { for all } s \in[0, T]
$$

and hence

$$
\beta(s)=\mu(s)-\rho(s) .
$$


This gives, under the conditions of Theorem 2.12,

$$
\pi^{*}(s)=\frac{\hat{\mu}(s)-\hat{\rho}(s)}{\hat{\sigma}^{2}(s)}
$$

b) In particular, if we assume that

$$
\mathcal{E}_{t}=\mathcal{F}_{t}=\mathcal{G}_{t} \quad \text { for all } t \in[0, T]
$$

then we get the well-known result

$$
\pi^{*}(s)=\frac{\mu(s)-\rho(s)}{\sigma^{2}(s)}
$$

and

$$
\Phi(x)=\log x+E\left[\int_{0}^{T}\left\{\rho(s)+\frac{1}{2}\left(\frac{\mu(s)-\rho(s)}{\sigma(s)}\right)^{2}\right\} d s\right]
$$

provided that

$$
E\left[\int_{0}^{T}\left(\frac{\mu(s)-\rho(s)}{\sigma(s)}\right)^{2} d s\right]<\infty
$$

\section{Example 2.14 (Delayed noise effect)}

Suppose $\mathcal{E}_{t}=\mathcal{F}_{t}$ and $\mathcal{G}_{t}=\mathcal{F}_{t+\delta}$ for some $\delta>0$. Let $\mu(s)$ and $\rho(s)$ be bounded $\mathcal{F}_{s+\delta^{-}}$ measurable and choose

$$
\sigma(s)=\exp (B(s+\delta)) ; \quad s \in[0, T] .
$$

(See Example 1.4). Then $D_{s^{+}} \sigma(s)=\sigma(s)$ and hence the corresponding optimal portfolio is, by Theorem 2.12 ,

$$
\pi_{\delta}^{*}(s)=\frac{E\left[\mu(s)-\rho(s)+\sigma(s) \mid \mathcal{F}_{s}\right]}{E\left[\sigma^{2}(s) \mid \mathcal{F}_{s}\right]} \quad \text { for } \delta>0 .
$$

On the other hand, if $\mathcal{E}_{t}=\mathcal{F}_{t}=\mathcal{G}_{t}$ (corresponding to $\delta=0$ ) then $D_{s^{+}} \sigma(s)=0$ and we know by Corollary 2.13 that the optimal portfolio is

$$
\pi_{0}^{*}(s)=\frac{\mu(s)-\rho(s)}{\sigma^{2}(s)} .
$$

Comparing (2.44) and (2.45) we see that, perhaps surprisingly,

$$
\lim _{\delta \rightarrow 0^{+}} \pi_{\delta}^{*}(s) \neq \pi_{0}^{*}(s)
$$


Similarly, if the corresponding value functions are denoted by $\Phi_{\delta}(s)$ and $\Phi_{0}(x)$, respectively, we get

$$
\lim _{\delta \rightarrow 0^{+}} \Phi_{\delta}(x)=\log x+E\left[\int_{0}^{T}\left\{\rho(s)+\frac{1}{2}\left(\frac{\mu(s)-\rho(s)}{\sigma(s)}+1\right)^{2}\right\} d s\right] \neq \Phi_{0}(x) .
$$

We conclude that any positive delay $\delta$ in the information, no matter how small, has a substantial effect on the optimal control and the value function.

\section{The pure jump case $(\sigma=0)$}

Referring to Example 1.2 we now consider the market $\mathcal{N}(\mathcal{E}, \mathcal{G})$ given by

$$
\begin{aligned}
& \text { (bond price) } \quad d S_{0}(t)=\rho(t) S_{0}(t) d t ; \quad S_{0}(0)=1 \\
& \text { (stock price) } \quad d S_{1}(t)=S_{1}\left(t^{-}\right)\left[\mu(t) d t+\int_{\mathbb{R}} \theta(t, z) \tilde{N}\left(d^{-} t, d z\right)\right] ; \quad S_{1}(0)>0
\end{aligned}
$$

where we assume that $\rho(t), \mu(t)$ and $\theta(t, z)$ satisfy the following conditions:

$\rho(t), \mu(t)$ and $\theta(t, z)$ are $\mathcal{G}_{t}$ - measurable, for all $t \in[0, T], z \in \mathbb{R}$

$\theta(t, z)$ is bounded and Malliavin differentiable and $D_{t^{+}, z} \theta(t, z):=\lim _{s \rightarrow t^{+}} D_{s, z} \sigma(t, z)$

exists for a.a. $t, z$ and is bounded, where $D_{s, z}$ denotes the Malliavin derivative at $s, z$ (see Definition 3.5)

$\mathrm{E}\left[\int_{0}^{T}\left\{|\rho(s)|+|\mu(s)|+\int_{\mathbb{R}}\left(|\theta(s, z)|+\left|D_{s^{+}, z} \theta(s, z)\right|\right) \nu(d z)\right\} d s\right]<\infty$, where $\nu$ is the Lévy measure of $\eta(\cdot)$, so that $\tilde{N}(d t, d z)=N(d t, d z)-\nu(d z) d t$

(3.6) The equation (3.2) has a unique $\mathcal{G}_{t}$-adapted solution $S_{1}(t) ; t \in[0, T]$

As before $\left\{\mathcal{E}_{t}\right\}_{t \in[0, T]}$ and $\left\{\mathcal{G}_{t}\right\}_{t \in[0, T]}$ are given filtrations such that

$$
\mathcal{E}_{t} \subseteq \mathcal{F}_{t} \subseteq \mathcal{G}_{t} \subseteq \mathcal{F} \quad \text { for all } t \in[0, T]
$$

Definition 3.1 The set $\mathcal{A}_{\mathcal{E}}$ of admissible portfolios consists of all processes $\pi(t)$ satisfying 
the following conditions:

$\pi(t)$ is $\mathcal{E}_{t}$-adapted

$\pi(t) \theta(t, z)$ is Skorohod integrable with respect to $\tilde{N}(\cdot, \cdot)$

(see Definition 3.4) and caglad.

$\pi(t) \theta(t, z)>-1+\epsilon$ for a.a. $t, z$ (where $\epsilon>0$ may depend on $\pi$ ), and

$E\left[\int_{0}^{T} \int_{\mathbb{R}}|\log (1+\pi(s) \theta(s, z))| \nu(d z) d t\right]<\infty$

$\pi(t)\left(\theta(t, z)+D_{t^{+}, z} \theta(t, z)\right)>-1+\epsilon$ for a.a. $t, z$ (where $\epsilon>0$ may depend

on $\pi)$, and

$E\left[\int_{0}^{T} \int_{\mathbb{R}}\left|\log \left(1+\pi(t)\left(\theta(t, z)+D_{t^{+}, z} \theta(t, z)\right)\right)\right| \nu(d z) d t\right]<\infty$.

Problem 3.2 Find $\Phi(x)$ and $\pi^{*} \in \mathcal{A}_{\mathcal{E}}$ such that

$$
\Phi(x)=\sup _{\pi \in \mathcal{A}_{\mathcal{E}}} E^{x}\left[\log X^{(\pi)}(T)\right]=E^{x}\left[\log \left(X^{\left(\pi^{*}\right)}(T)\right)\right]
$$

where $X^{(\pi)}(t)=X(t)$ is given by $X(0)=x>0$ and

$$
d X(t)=X\left(t^{-}\right)\left[(\rho(t)+(\mu(t)-\rho(t)) \pi(t)) d t+\pi(t) \int_{\mathbb{R}} \theta(t, z) \tilde{N}\left(d^{-} t, d z\right)\right] .
$$

The function $\Phi \leq \infty$ is called the value function and $\pi^{*}$ (if it exists) is called an optimal portfolio for Problem 3.2.

Before studying Problem 3.2 more closely we review some mathematical background about Malliavin calculus and anticipating calculus for jump diffusions. For proof and details we refer to [DMØP1]. See also [DMØP2] for other, related applications.

First we recall the chaos expansion in terms of iterated integrals with respect to the compensated Poisson random measure $\tilde{N}(d t, d z)$, originally due to [I]. (See also [L].)

Let $\lambda$ denote Lebesgue measure on $[0, T]$ and let $L^{2}\left((\lambda \times \nu)^{n}\right)$ be the space of all deterministic functions $f:([0, T] \times \mathbb{R})^{n} \rightarrow \mathbb{R}$ such that

$$
\|f\|_{L^{2}\left((\lambda \times \nu)^{n}\right)}^{2}:=\int_{([0, T] \times \mathbb{R})^{n}} f^{2}\left(t_{1}, z_{1}, \ldots, t_{n}, z_{n}\right) d t_{1} \nu\left(d z_{1}\right) \ldots d t_{n} \nu\left(d z_{n}\right)<\infty .
$$

If $f$ is a real function on $([0, T] \times \mathbb{R})^{n}$ we define its symmetrization $\tilde{f}$ with respect to the variables $\left(t_{1}, z_{1}\right), \ldots,\left(t_{n}, z_{n}\right)$ by

$$
\tilde{f}\left(t_{1}, z_{1}, \ldots, t_{n}, z_{n}\right)=\frac{1}{n !} \sum_{\sigma} f\left(t_{\sigma_{1}}, z_{\sigma_{1}}, \ldots t_{\sigma_{n}}, z_{\sigma_{n}}\right)
$$


where the sum is taken over all permutations $\sigma$ of $\{1, \ldots, n\}$. We say that $f$ is symmetric if $\tilde{f}=f$ and we let $\tilde{L}^{2}\left((\lambda \times \nu)^{n}\right)$ denote the set of all symmetric functions in $L^{2}\left((\lambda \times \nu)^{n}\right)$. Put

$$
G_{n}=\left\{\left(t_{1}, z_{1}, \ldots, t_{n}, z_{n}\right) ; 0 \leq t_{1} \leq \cdots \leq t_{n} \leq T \quad \text { and } \quad z_{i} \in \mathbb{R}, i=1, \ldots, n\right\}
$$

and let $L^{2}\left(G_{n}\right)$ be the set of functions $g: G_{n} \rightarrow \mathbb{R}$ such that

$$
\|g\|_{L^{2}\left(G_{n}\right)}^{2}:=\int_{G_{n}} g^{2}\left(t_{1}, z_{1}, \ldots, t_{n}, z_{n}\right) d t_{1} \nu\left(d z_{1}\right) \ldots d t_{n} \nu\left(d z_{n}\right)<\infty .
$$

Note that

$$
\|f\|_{L^{2}\left((\lambda \times \nu)^{n}\right)}^{2}=n !\|f\|_{L^{2}\left(G_{n}\right)}^{2} ; \quad f \in \tilde{L}^{2}\left((\lambda \times \nu)^{n}\right) .
$$

If $f \in L^{2}\left(G_{n}\right)$ we define its $n$-fold iterated integral with respect to $\tilde{N}(\cdot, \cdot)$ by

$$
J_{n}(f)=\int_{0}^{T} \int_{\mathbb{R}} \ldots \int_{0}^{t_{2}} \int_{\mathbb{R}} f\left(t_{1}, z_{1}, \ldots, t_{n}, z_{n}\right) \tilde{N}\left(d t_{1}, d z_{1}\right) \ldots \tilde{N}\left(d t_{n}, d z_{n}\right)
$$

and if $f \in \tilde{L}^{2}\left((\lambda \times \nu)^{n}\right)$ we define

$$
I_{n}(f):=\int_{([0, T] \times \mathbb{R})^{n}} f\left(t_{1}, z_{1}, \ldots, t_{n}, z_{n}\right) \tilde{N}^{\otimes n}(d t, d z):=n ! J_{n}(f) .
$$

Then we have the following chaos expansion theorem:

Theorem $3.3([\mathbf{I}],[\mathbf{L}]) \quad$ Every $\mathcal{F}_{T}$-measurable random variable $F \in L^{2}(P)$ can be written

$$
F=E[F]+\sum_{n=1}^{\infty} I_{n}\left(f_{n}\right):=\sum_{n=0}^{\infty} I_{n}\left(f_{n}\right)
$$

for a unique sequence of deterministic functions $f_{n} \in \tilde{L}^{2}\left((\lambda \times \nu)^{n}\right)$. Moreover, we have the isometry

$$
E\left[F^{2}\right]=(E[F])^{2}+\sum_{n=1}^{\infty} n !\left\|f_{n}\right\|_{L^{2}\left((\lambda \times \nu)^{n}\right)}^{2} .
$$

Using this expansion theorem we can now define Skorohod integration and Malliavin differentiation as follows:

Definition 3.4 Let $\phi(t, z, \omega):[0, T] \times \mathbb{R} \times \Omega \rightarrow \mathbb{R}$ be a random field such that

$$
E\left[\phi^{2}(t, z)\right]<\infty
$$


and

$$
\phi(t, z, \cdot) \quad \text { is } \mathcal{F}_{T} \text {-measurable, for all }(t, z) \in[0, T] \times \mathbb{R} \text {. }
$$

Let

$$
\phi(t, z)=\sum_{n=0}^{\infty} I_{n}\left(f_{n}(\cdot, t, z)\right)
$$

be the chaos expasion of $\phi(t, z, \cdot)$, as given by Theorem 3.3. Let $\tilde{f}_{n}\left(t_{1}, z_{1}, \ldots, t_{n}, z_{n}, t_{n+1}, z_{n+1}\right)$ be the symmetrization of $f_{n}\left(t_{1}, z_{1}, \ldots, t_{n}, z_{n}, t, z\right)$ as a function of the $n+1$ variables $\left(t_{1}, z_{1}\right)$, $\left(t_{2}, z_{2}\right), \ldots\left(t_{n}, z_{n}\right)$ and $\left(t_{n+1}, z_{n+1}\right)=(t, z)$. Suppose that

$$
\sum_{n=0}^{\infty}(n+1) !\left\|\tilde{f}_{n}\right\|_{L^{2}\left((\lambda \times \nu)^{n}\right)}^{2}<\infty .
$$

Then the Skorohod integral of $\phi$ with respect to $\tilde{N}$ is defined by

$$
\int_{0}^{T} \int_{\mathbb{R}} \phi(t, z) \tilde{N}(\delta t, d z)=\sum_{n=0}^{\infty} I_{n+1}\left(\tilde{f}_{n}\right) .
$$

Note that

$$
E\left[\left(\int_{0}^{T} \int_{\mathbb{R}} \phi(t, z) \tilde{N}(\delta t, d z)\right)^{2}\right]=\sum_{n=0}^{\infty}(n+1) !\left\|\tilde{f}_{n}\right\|_{L^{2}\left((\lambda \times \nu)^{n}\right)}^{2}
$$

so the Skorohod integral of $\phi$ belongs to $L^{2}(P)$ if it exists. Moreover,

$$
E\left[\int_{0}^{T} \int_{\mathbb{R}} \phi(t, z) \tilde{N}(\delta t, d z)\right]=0 .
$$

The Skorohod integral with respect to a Poisson random measure was first constructed by Y. Kabanov [K1], [K2]. It is is an extension of the Itô integral in the sense that if $\phi(t, z)$ is assumed to be $\mathcal{F}_{t}$-measurable for all $(t, z) \in[0, T] \times \mathbb{R}$, then the two integrals coincide:

$$
\int_{0}^{T} \int_{\mathbb{R}} \phi(t, z) \tilde{N}(\delta t, d z)=\int_{0}^{T} \int_{\mathbb{R}} \phi(t, z) \tilde{N}(d t, d z) .
$$

(See also [DØP, Prop. 3.2].)

Definition 3.5 Let $F \in L^{2}(P)$ be $\mathcal{F}_{T}$-measurable, with the expansion

$$
F=\sum_{n=0}^{\infty} I_{n}\left(f_{n}\right) ; \quad f_{n} \in \tilde{L}^{2}\left((\lambda \times \nu)^{n}\right) .
$$


Suppose $F \in \mathbb{D}_{1,2}$, i.e.

$$
\|F\|_{\mathbb{D}_{1,2}}^{2}:=(E[F])^{2}+\sum_{n=1}^{\infty} n n !\left\|f_{n}\right\|_{L^{2}\left((\lambda \times \nu)^{n}\right)}^{2}<\infty .
$$

Then we define the Malliavin derivative (or stochastic derivative) of $F$ at $(t, z) \in[0, T] \times \mathbb{R}$ by

$$
D_{t, z} F:=\sum_{n=1}^{\infty} n I_{n-1}\left(f_{n}(\cdot, t, z)\right)
$$

where $I_{n-1}\left(f_{n}(\cdot, t, z)\right)$ means that we perform the $n-1$ iterated integral with respect to the first $n-1$ variable pairs $\left(t_{1}, z_{1}\right), \ldots,\left(t_{n-1}, z_{n-1}\right)$ and put $\left(t_{n}, z_{n}\right)=(t, z)$.

Using the isometry

$$
E\left[\int_{0}^{T} \int_{\mathbb{R}}\left(D_{t, z} F\right)^{2} \nu(d z) d t\right]=\sum_{n=1}^{\infty} n n !\left\|f_{n}\right\|_{L^{2}\left((\lambda \times \nu)^{n}\right)}^{2}<\infty,
$$

we see that if (3.22) holds, then $D_{t, z} F$ exists for a.a. $(t, z) \in[0, T] \times \mathbb{R}$ with respect to $\lambda \times \nu$.

In the pure jump case the Malliavin derivative $D=D_{t, z}$ is a difference operator, in the sense that it satisfies the following product rule

$$
D(F \cdot G)=F \cdot D G+G \cdot D F+D F \cdot D G,
$$

if both $F$ and $G$ are Malliavin differentiable random variables. (See [DØP, Lemma 3.9] and [L].)

From this we get the following result:

Lemma 3.6 Let $f: \mathbb{R} \rightarrow \mathbb{R}$ be continuous and let $F$ be a Malliavin differentiable random variable. Then, with $D=D_{t, z}$,

$$
D(f(F))=f(F+D F)-f(F) .
$$

Proof. By (3.24) we get that

$$
D\left(F^{2}\right)=2 F \cdot D F+D F \cdot D F=(F+D F)^{2}-F^{2}
$$

and by induction

$$
D\left(F^{n}\right)=(F+D F)^{n}-F^{n} .
$$

Hence (3.25) holds for all polynomials $f$ and hence for all continuous functions $f$ with compact support, by the Weierstrass approximation theorem. The result then follows by a limit argument, using the closedness of $D_{t, z}$.

We now turn to the definition of a forward integral with respect to $\tilde{N}(\cdot, \cdot)$. (Compare with Definition 2.7.) 
Definition 3.7 ([DMØP1]) The forward integral of a random field $\phi(t, z)=\phi(t, z, \omega)$ with respect to $\tilde{N}(\cdot, \cdot)$ is defined by

$$
\int_{0}^{T} \int_{\mathbb{R}} \phi(t, z, \omega) \tilde{N}\left(d^{-} t, d z\right)=\lim _{m \rightarrow \infty} \int_{0}^{T} \int_{K_{m}} \phi(t, z) \tilde{N}(d t, d z)
$$

if the limit exists in probability. Here $\left\{K_{m}\right\}_{m=1}^{\infty}$ is an increasing sequence of compact sets in $\mathbb{R} \backslash\{0\}$ such that

$$
\mathbb{R} \backslash\{0\}=\bigcup_{m=1}^{\infty} K_{m} \quad \text { and } \quad \nu\left(K_{m}\right)<\infty \quad \text { for all } m .
$$

Just as in the continuous case (Lemma 2.8) we have

Lemma 3.8 Suppose that $t \rightarrow \phi(t, z, \omega)$ is caglad for a.a. $z, \omega$ with respect to $\nu \times P$ and that $\phi(t, z, \cdot)$ is $\mathcal{G}_{t}$-measurable for all $t \in[0, T]$ and a.a. $z$ with respect to $\nu$. Moreover, assume that $\eta(t)$ is a semimartingale with respect to $\mathcal{G}_{t}$. Then if $\phi$ is forward integrable with respect to $\tilde{N}$ we have

$$
\int_{0}^{T} \int_{\mathbb{R}} \phi(t, z, \omega) \tilde{N}\left(d^{-} t, d z\right)=\int_{0}^{T} \int_{\mathbb{R}} \phi(t, z, \omega) \tilde{N}(d t, d z),
$$

where the integral on the right is the usual semimartingale integral.

Here is a relation between forward integrals and Skorohod integrals (compare with Lemma 2.9):

\section{Lemma 3.9 ([DMØP1, Lemma 4.3])}

If the forward integral of $\phi$ exists in $L^{2}(P)$ then

$$
\begin{aligned}
& \int_{0}^{T} \int_{\mathbb{R}} \phi(t, z) \tilde{N}\left(d^{-} t, d z\right) \\
& \quad=\int_{0}^{T} \int_{\mathbb{R}} D_{t^{+}, z} \phi(t, z) \nu(d z) d t+\int_{0}^{T} \int_{\mathbb{R}}\left(\phi(t, z)+D_{t^{+}, z} \phi(t, z)\right) \tilde{N}(\delta t, d z),
\end{aligned}
$$

provided that

$$
D_{t^{+}, z} \phi(t, z)=\lim _{s \rightarrow t^{+}} D_{s, z} \phi(t, z)
$$

exists and is integrable with respect to $\lambda \times \nu$. 
Lemma 3.10 Let $\phi$ be as in Lemma 3.9. Then

$$
E\left[\int_{0}^{T} \int_{\mathbb{R}} \phi(t, z) \tilde{N}\left(d^{-} t, d z\right)\right]=E\left[\int_{0}^{T} \int_{\mathbb{R}} D_{t^{+}, z} \phi(t, z) \nu(d z) d t\right],
$$

provided the integrals exist.

Finally we state an Itô formula for forward integrals with respect to $\tilde{N}(\cdot, \cdot)$ (compare with Theorem 2.11):

Theorem 3.11 ([DMØP1]) Let $X(t)$ be a process of the form

$$
X(t)=x+\int_{0}^{t} \alpha(s) d s+\int_{0}^{t} \int_{\mathbb{R}} \theta(s, z) \tilde{N}\left(d^{-} s, d z\right)
$$

and let $f \in C^{2}(\mathbb{R})$. Then

$$
\begin{aligned}
& f(X(t))=f(x)+\int_{0}^{t} f^{\prime}(X(s)) \alpha(s) d s \\
& +\int_{0}^{t} \int_{\mathbb{R}}\left\{f\left(X\left(s^{-}\right)+\theta(s, z)\right)-f\left(X\left(s^{-}\right)\right)-f^{\prime}\left(X\left(s^{-}\right)\right) \theta(s, z)\right\} \nu(d z) d t \\
& +\int_{0}^{t} \int_{\mathbb{R}}\left\{f\left(X\left(s^{-}\right)+\theta(s, z)\right)-f\left(X\left(s^{-}\right)\right)\right\} \tilde{N}\left(d^{-} s, d z\right) .
\end{aligned}
$$

provided that at least one of the integrals converges.

We now have the necessary mathematical machinery for solving Problem 3.2. First note that if we apply the Itô formula for forward integrals (Theorem 3.11), we get that the solution of equation (3.13) is given by

$$
\begin{aligned}
X(t)=x & \exp \left[\int_{0}^{t}\{\rho(s)+(\mu(s)-\rho(s)) \pi(s)\right. \\
& \left.+\int_{\mathbb{R}}[\log (1+\pi(s) \theta(s, z))-\pi(s) \theta(s, z)] \nu(d z)\right\} d s \\
& \left.+\int_{0}^{t} \int_{\mathbb{R}} \log (1+\pi(s) \theta(s, z)) \tilde{N}\left(d^{-} s, d z\right)\right] .
\end{aligned}
$$


(See e.g. Example 1.2.2 in [ØS].) Hence, using Lemma 3.10 we get

$$
\begin{aligned}
& E\left[\log \frac{X(T)}{x}\right]=E\left[\int_{0}^{T}\{\rho(s)+(\mu(s)-\rho(s)) \pi(s)\right. \\
& \left.\quad+\int_{\mathbb{R}}[\log (1+\pi(s) \theta(s, z))-\pi(s) \theta(s, z)] \nu(d z)\right\} d s \\
& \left.\quad+\int_{0}^{T} \int_{\mathbb{R}} \log (1+\pi(s) \theta(s, z)) \tilde{N}\left(d^{-} s, d z\right)\right] \\
& =E\left[\int _ { 0 } ^ { T } \left\{\rho(s)+(\mu(s)-\rho(s)) \pi(s)+\int_{\mathbb{R}}[\log (1+\pi(s) \theta(s, z))-\pi(s) \theta(s, z)\right.\right. \\
& \left.\left.\left.+D_{s^{+}, z} \log (1+\pi(s) \theta(s, z))\right] \nu(d z)\right\} d s\right]=: F(\pi) .
\end{aligned}
$$

By Lemma 3.6 we get

$$
\begin{aligned}
D_{s^{+}, z} & \log (1+\pi(s) \theta(s, z)) \\
& =\log \left(1+\pi(s) \theta(s, z)+D_{s^{+}, z}(\pi(s) \theta(s, z))\right)-\log (1+\pi(s) \theta(s, z)) \\
& =\log \left(1+\pi(s)\left(\theta(s, z)+D_{s^{+}, z} \theta(s, z)\right)-\log (1+\pi(s) \theta(s, z))\right. \\
& =\log \left(1+\frac{\pi(s) D_{s^{+}, z} \theta(s, z)}{1+\pi(s) \theta(s, z)}\right)
\end{aligned}
$$

Substituted into (3.35) this gives

$$
\begin{aligned}
F(\pi) & :=E\left[\int_{0}^{T}\{\rho(s)+(\mu(s)-\rho(s)) \pi(s)\right. \\
& \left.\left.+\int_{\mathbb{R}}\left[\log \left(1+\pi(s)\left(\theta(s, z)+D_{s^{+}, z} \theta(s, z)\right)\right)-\pi(s) \theta(s, z)\right] \nu(d s)\right\} d s\right] .
\end{aligned}
$$

We want to maximize the function

$$
\pi \rightarrow F(\pi) ; \quad \pi \in \mathcal{A}_{\mathcal{E}}
$$

Suppose that an optimal $\pi^{*} \in \mathcal{A}_{\mathcal{E}}$ exists. Then for all bounded $\eta \in \mathcal{A}_{\mathcal{E}}$ there exists $\delta>0$ such that $\pi^{*}+r \eta \in \mathcal{A}_{\mathcal{E}}$ for $r \in(-\delta, \delta)$ and the function

$$
f(r):=F\left(\pi^{*}+r \eta\right) ; \quad r \in(-\delta, \delta)
$$


is maximal for $r=0$. Therefore

$$
\begin{aligned}
0= & f^{\prime}(0)=E\left[\int_{0}^{T}\{(\mu(s)-\rho(s)) \eta(s)\right. \\
& \left.\left.+\int_{\mathbb{R}}\left[\left(1+\pi^{*}(s) \tilde{\theta}(s, z)\right)^{-1} \tilde{\theta}(s, z) \eta(s)-\theta(s, z) \eta(s)\right] \nu(d z)\right\} d s\right]
\end{aligned}
$$

where we have put

$$
\tilde{\theta}(s, z)=\theta(s, z)+D_{s^{+}, z} \theta(s, z)
$$

Hence

$$
\int_{0}^{T} E\left[\left\{\mu(s)-\rho(s)+\int_{\mathbb{R}}\left[\left(1+\pi^{*}(s) \tilde{\theta}(s, z)\right)^{-1} \tilde{\theta}(s, z)-\theta(s, z)\right] \nu(d z)\right\} \eta(s)\right] d s=0 .
$$

Since for each $s$ the random variables $\eta(s) ; \eta \in \mathcal{A}_{\mathcal{E}}$, generate the whole $\sigma$-algebra $\mathcal{E}_{s}$, we conclude that, for all $s \in[0, T]$,

$$
E\left[\left\{\mu(s)-\rho(s)+\int_{\mathbb{R}}\left[\left(1+\pi^{*}(s) \tilde{\theta}(s, z)\right)^{-1} \tilde{\theta}(s, z)-\theta(s, z)\right] \nu(d z)\right\} \mid \mathcal{E}_{s}\right]=0 .
$$

This proves part a) of the following result:

Theorem 3.12 a) Suppose there exists an optimal portfolio $\pi^{*} \in \mathcal{A}_{\mathcal{E}}$ for Problem 3.2. Then $y=\pi^{*}(s)$ satisfies the equation

$$
\begin{aligned}
& E\left[\int_{\mathbb{R}} \frac{\theta(s, z)+D_{s^{+}, z} \theta(s, z)}{1+y\left(\theta(s, z)+D_{s^{+}, z} \theta(s, z)\right)} \nu(d z) \mid \mathcal{E}_{s}\right] \\
& \quad=E\left[\left\{\rho(s)-\mu(s)+\int_{\mathbb{R}} \theta(s, z) \nu(d z)\right\} \mid \mathcal{E}_{s}\right] ; \quad s \in[0, T] .
\end{aligned}
$$

b) Suppose

$$
\theta(s, z)+D_{s^{+}, z} \theta(s, z) \geq 0 \quad \text { for a.a. } s, z
$$

and that for all $s$ there exists a solution

$$
y=: \hat{\pi}(s)
$$

of equation (3.41). Suppose

$$
\hat{\pi}(s) \in \mathcal{A}_{\mathcal{E}} .
$$

Then $\hat{\pi}$ is an optimal portfolio for Problem 3.2. 
Proof of b): If (3.42) holds, then the function $F(\pi)$ given by (3.37) is concave.

Example 3.13 (The Poisson process) Suppose $\eta(t)$ is a compensated Poisson process. Then the Lévy meassure $\nu(d z)$ is the point mass at $z=1$ and (3.41) gets the form

$$
E\left[\frac{\tilde{\theta}(s)}{1+y \tilde{\theta}(s)} \mid \mathcal{E}_{s}\right]=E\left[\rho(s)-\mu(s)+\theta(s, 1) \mid \mathcal{E}_{s}\right]
$$

where

$$
\tilde{\theta}(s)=\theta(s, 1)+D_{s^{+}, 1} \theta(s, 1)
$$

Assume in addition that

$$
\tilde{\theta}(s) \text { is } \mathcal{E}_{s} \text {-measurable. }
$$

Then (3.43) has the solution

$$
y=\hat{\pi}(s)=\pi^{*}(s)=E\left[\rho(s)-\mu(s)+\theta(s, 1) \mid \mathcal{E}_{s}\right]^{-1}-(\tilde{\theta}(s))^{-1},
$$

provided that

$$
E\left[\rho(s)-\mu(s)+\theta(s, 1) \mid \mathcal{E}_{s}\right] \neq 0 \quad \text { and } \quad \tilde{\theta}(s) \neq 0 ; s \in[0, T] .
$$

Corollary 3.14 (Complete information case) Supppose

$$
\mathcal{E}_{t}=\mathcal{F}_{t}=\mathcal{G}_{t} \quad \text { for all } t \in[0, T]
$$

and that there exists an optimal portfolio $\pi^{*} \in \mathcal{A}_{\mathcal{E}}$ for Problem 3.2. Then $y=\pi^{*}(s)$ solves the equation

$$
\int_{\mathbb{R}} \frac{\theta(s, z)}{1+y \theta(s, z)} \nu(d z)=\rho(s)-\mu(s)+\int_{\mathbb{R}} \theta(s, z) \nu(d z) .
$$

In the special case of Markovian coefficients this result could have been obtained by dynamic programming.

\section{References}

[BL] F.E. Benth and A. Løkka: Anticipative calculus for Lévy processes and stochastic differential equations. Preprint Series, Dept. of Mathematics, Univ. of Oslo, 6/2002.

[BØ] F. Biagini and B. Øksendal: A general stochastic calculus approach to insider trading. Preprint Series, Dept. of Mathematics, Univ. of Oslo, 17/2002. 
[DMØP1] G. Di Nunno, B. Øksendal and F. Proske: Malliavin calculus and anticipative Itô formulae for Lévy processes. Preprint Series, Dept. of Mathematics, Univ. of Oslo, $16 / 2003$.

[DMØP2] G. Di Nunno, T. Meyer-Brandis, B. Øksendal and F. Proske: Optimal portfolio for an insider in a market driven by Lévy processes. Preprint Series, Dept. of Mathematics, Univ. of Oslo, 36/2003.

[HØ] Y. Hu and B. Øksendal: Optimal smooth portfolio selection for an insider. Preprint Series, Dept. of Mathematics, Univ. of Oslo 12/2003.

[I] K. Itô: Spectral type of the shift transformation of differential processes with stationary increments. Trans. Amer. Math. Soc 81 (1956), 253-263.

[K1] Y. Kabanov: A generalized Itô formula for an extended stochastic integral with respect to a Poisson random measure. (In Russian) Usp. Mat. Nauk 29 (1974), No. 4 (178), pp. $167-168$.

[K2] Y. Kabanov: On extended stochastic intervals. Theory of Probab. and its Appl. 20 (1975), 710-722.

[L] A. Løkka: Martingale representations and functionals of Lévy processes. Preprint Series, Dept. of Mathematics, Univ. of Oslo, 21/2001.

[N] D. Nualart: The Malliavin Calculus and Related Topics. Springer-Verlag 1995.

[NP] D. Nualart and E. Pardoux: Stochastic calculus with anticipating integrands. Probab. Th. Rel. Fields 78 (1988), 555-581.

[Ø] B. Øksendal: An Introduction to Malliavin Calculus with Applications to Economics. Working Paper 3/1996, Norwegian School of Economics and Business Administration.

[ØS] B. Øksendal and A. Sulem: Applied Stochastic Control of Jump Diffusions. SpringerVerlag 2004 (forthcoming book).

[RV1] F. Russo and P. Vallois: Forward, backward and symmetric stochastic integration. Probab. Th. Rel. Fields 97 (1993), 403-421.

[RV2] F. Russo and P. Vallois: Stochastic calculus with respect to continuous finite quadratic variation processes. Stochastics and Stochastics Reports 70 (2000), 1-40. 